\title{
Antibacterial and Wound Healing Potential of Ethanolic Extract of Zingiber Officinale in Albino Rats
}

\author{
Aisha Hassan Balla Mohamed ${ }^{1,}$, Amira Altom Fawzi Osman ${ }^{2}$ \\ ${ }^{1}$ Microbiology Department, Alzaiem Alazhari University (AAU), Khartoum, Sudan \\ ${ }^{2}$ Microbiology Department, Faculty of Medical Laboratories, Alzaiem Alazhari University (AAU), Khartoum, Sudan \\ Email address: \\ Jojohassan51513@gmail.com (A. H. B. Mohamed), Amiratom2014@gmail.com (A. A. F. Osman) \\ ${ }^{*}$ Corresponding author
}

\section{To cite this article:}

Aisha Hassan Balla Mohamed, Amira Altom Fawzi Osman. Antibacterial and Wound Healing Potential of Ethanolic Extract of Zingiber Officinale in Albino Rats. Journal of Diseases and Medicinal Plants. Vol. 3, No. 1, 2017, pp. 1-6. doi: 10.11648/j.jdmp.20170301.11

Received: November 25, 2016; Accepted: January 3, 2017; Published: January 24, 2017

\begin{abstract}
Medicinal plants are used in a wide range in Sudan to treat several diseases, Zingiber officinale Roscoe is one of the plants that are central to the lives of traditional societies in Africa it has been reported as traditional folkloric medicine, the plant roots are used in some parts in Sudan for wound healing, this study aimed to assess the antibacterial and wound healing activity of Zingiber officinale ethanolic extract in wound infected albino rats and compare the effect of the plant against commercial reference drug against Staphylococcus aureus, methods of this study included fifteen swiss wistar albino rat, divided into three groups of five rats (Group 1 (wounded +infection, Group 2 wound +infection+ fusiderm ointment, Group 3 wound + infection $+12 \%$ ethanol extract of Zingiber officinale with soft yellow paraffin), all results included microbial examination for bacterial count, histological examination for the presence of inflammatory cells and collagen and healing percentage measured by transparent ruler, the results of in vitro antimicrobial activity of the extract gave clear zones of inhibition on the standard strains of Proteus mirabilis, Klebsiellia pneumoniae, Escherichia coli, Pseudomonas aeruginosa and Staphylococcus aureus, significant reduction of bacterial number observed in ginger treated group from. $5 \times 10^{8} \mathrm{cfu} / \mathrm{ml}$ to. $5 \times 10^{2}$ $\mathrm{cfu} / \mathrm{ml}$ on day 8 of dressing while the positive control group showed bacterial count reduction from. $5 \times 10^{8} \mathrm{cfu} / \mathrm{ml}$ to complete diminishing of bacterial infection. Significant difference in the wound closure was observed in positive control group (fusiderm) to give a percentage of $55.7 \%$ on day 4 and $91 \%$ on day 8 . The ginger treatment group also showed faster wound healing with a percentage closed to positive control $51.4 \%$ on day 4 and $90.6 \%$ on day 8 , while the wound healing rate was slow and took more than 8 days in negative control, histological examination of granulated tissue done by Haematoxylin and Eosin stain and van Gieson stain showed more collagen fibers formation and reduction of inflammatory cell during the healing period, finally the study concluded that Zingiber officinale ethanolic extract exert antibacterial and wound healing capacity, this study established a good support to the use of plants in herbal medicine and as abase for the development of new drugs.
\end{abstract}

Keywords: Wound Infection, Zingiber Officinale, Ginger, Anti-bacterial Activity, Healing Activity

\section{Introduction}

Wounds generally termed as physical injuries that result in an opening or breaking of the skin, there are different types of wounds which range from mild to potentially fatal, wound infections are most common in developing countries, such as Sub-Saharan African and south Asian, than in developed countries, current estimates indicate that nearly 6 million people suffer from chronic wounds worldwide [1], the poor hygienic condition in some third world is the main cause of this problem, besides that most of the people in developing countries suffer from an infected wound cannot afford to buy modern drugs, which are very high costing and might have side effects. When the subcutaneous tissue become exposed by the wound that provides a moist, warm and nutritionally rich environment, then of the microbial colonization and proliferation occurs, since group of potential pathogenic microbes exist in colonization, any wound is at some risk to become infected $[2,3]$, then most infection fail to heal, the patient suffer from more pain and trauma, high cost of treatment of the infected wound and more demanding of 
management practise $[4,5]$.

Herbs and spices are very important and useful as therapeutic agent against many pathological infection, a large number of plants are used for the treatment of various ailment, the medical value of those plants was recognized since ancient times, there are several natural products obtained from plants have offered a lead to the development of various pharmaceuticals uses [6], the medicinal benefit come from their phytochemical compounds including the isoflavones, anthocyanins and flavonoids there were found associated with the spices [7], they were also extensively investigated for their activity against a number of bacteria, fungi, plants, animals and human pathogen, insects, pests, and other harmful microorganisms [8].

Zingiber officinale Roscoe is a common spice originated from South east Asia; a city with its San- skrit name Shunti was already in existence in 200 B. C. Ginger is also called the Great Medicament" in Ayurvedic medicines, it belongs to Zingiberaceae family and is a perennial plant with thick tuberous rhizomes which are the medicinally useful part of this plant, medicinal history of ginger has been extensively searched throughout the world and found to possess antiinflammatory, cholesterol-lowering, and antithrombotic properties [9]. Important secondary metabolites present in the rhizome are curcumene, non-volatile hydroxyaryl compounds e.g. zingerone, gingeroles and shogaoles (phenylalkanones), volatile sesquiterpenes (e.g. zingiberene and bisabolene) and monoterpenoids (e.g. citral) [10], ginger may also decrease joints pain from arthritis, may have blood thinning and cholesterol lowering properties and may be useful for the treatment of heart diseases and lungs diseases, the odour and flavour of ginger root is caused by a mixture of gingerone, shoagoles and gingerols, volatile oils that make up about 1$3 \%$ of the weight fresh ginger, medically ginger is used as a stimulant and carminative, and is also used frequently for drypepsia and colic, it has a sialagogue action, stimulating the production of saliva, it is also used to disguise the taste of medicines. Ginger promotes the release of bile from the gall bladder [11].

\section{Material and Methods}

\subsection{Plant Material}

Samples of dry Zingiber officinale roots used in this study were bought from traditional sealer At a local market in Bahri State (Sudan), the dry plant material was milled using mortar. The powder obtained was used for extraction.

\subsection{Preparation of Ethanolic Extract}

The solvent used in the study is $95 \%$ ethanol the extraction of the ginger powder was carried out by methods soxhlet device, $90 \mathrm{gm}$ of ginger dissolved in $450 \mathrm{ml}$ of $95 \%$ ethanol the extraction lasted for 4 hours by heating the solution then concentrated using water bath at $100^{\circ} \mathrm{C}$ by rotative evaporator (Rota vapour). Then the conical flask was rotated in the water bath linked with source of low temperature to convert it to powder and returning the solvent for other use, then the extract left to dry for 2 days [12].

\subsection{In Vitro Antibacterial Activity}

\subsubsection{Microorganisms}

Its importance to determine the effectiveness of the plant extract against the standard bacteria that cause wound infection, standard strains have been obtained from the American Type Culture Collection: Staphylococcus aureus, Proteus mirabilis, Klebsiellia pneumoniae, Escherichia coli and Pseudomonas aeruginosa.

The isolates were sub-cultured into nutrient agar medium and incubated at $37^{\circ} \mathrm{C}$ for 24 hour prior to antibacterial testing using cup- plate agar diffusion method.

\subsubsection{Cup- Plate Agar Diffusion Method}

$0.2 \mathrm{ml}$ of the tested organisms inoculated into sterile Petri dishes, about $20 \mathrm{ml}$ of sterile mueller hunton media was poured into each dish, the dishes were gently rocked, then the media allowed to solidify, wells were dug into the media with sterile core borer of (NO.4) $10 \mathrm{~mm}$ in diameter, $0.1 \mathrm{ml}$ of extract introduced to the wells with different concentrations, then all dishes left standing for one hour for the proper diffusion and incubated at $37^{\circ} \mathrm{C}$ for 24 hour, zone of inhibition around the wells was measured to the nearest millimetre using transparent ruler [13].

\subsubsection{Minimum Inhibitory Concentration (MIC)}

The minimum inhibitory concentration of the extract was determined according to the macro broth dilution methods. Standardized suspension of the organisms $(200 \mu \mathrm{l})$ was inoculated into series of sterile tubes of nutrient broth containing two-fold dilutions of extract and incubated at $37^{\circ} \mathrm{C}$ for 24 hours. The minimum inhibitory concentrations were read as the least concentration that inhibited the growth of the test organisms [14].

\subsection{Wound Healing and Antimicrobial Activity}

\subsubsection{Ointment Formulation}

A $12 \%(w / w)$ Zingiber officinale ethanol extract prepared by mixing the extract $(6 \mathrm{~g})$ in yellow soft paraffin $(50 \mathrm{~g})$ obtained from a pharmacy, the mixture put in water path to dissolve the yellow soft paraffin and mixed gently in order to become homogenize [15].

\subsubsection{Animals}

Fifteen Swiss Wistar Albino rats of either sex, weighing $60-95 \mathrm{~g}$ were used. Animals were supplied by faculty of veterinary, university of Khartoum, Sudan. Animal housing and protocol approved by the Pharmacology Laboratory of Medicinal and Aromatic Plant Research Institute. National Centre for Research, Khartoum, Sudan.

Rats were housed in a ventilated animal house before and after surgery. They had access to food, the holding room was illuminated with 12 hours. Light/dark cycles. Room temperature was between $30-35^{\circ} \mathrm{C}$ with $45 \%$ to $55 \%$ humidity. 


\subsubsection{In Vivo Wound Healing}

Full thickness wounds $(1 \times 1) \mathrm{cm}$ were made in the skin of the tested animals, hair of the lower back and right flank of animals was fully shaved, the animals were held in standard crouching position, and the mobile skin of flank was gently stretched and held by fingers. A metal circular object measuring $1 \mathrm{~cm}$ in diameter was placed on stretched skin and an outline of the object was traced on the skin using a fine tipped pen.

The wound was made by excising the skin within the border of the object to level of loose subcutaneous tissue, using sterile forceps and scalpel blade. The artificial wounds were inoculated with $0.1 \mathrm{ml}$ of Staphylococcus aureus (ATCC25923), the suspension compared with McFarland to give $10^{8} \mathrm{cfu} / \mathrm{ml}$, after $24 \mathrm{hr}$ the animals divided into three groups, the ginger group applied with $12 \%$ ointment of the extract, negative control group left without treatment while the positive control group applied with commercial fusiderm ointment once daily for 8 days.

Healing percentage in a certain day was the difference between the initial wound (in day zero) and healing wound on that day [16].

$$
\begin{gathered}
\% \text { of wound closure }=\frac{\text { Wound area on day } 0-\text { Wound area on day } n}{\text { Wound area on day } 0} \times 100 \\
\text { n: number of days (4th and } 8 \text { th). }
\end{gathered}
$$

\subsubsection{In vivo antimicrobial activity}

Antimicrobial activity carried out using spectrophotometer (turbidity) method, samples were collected from the wound by sterile swab then transferred into $1 \mathrm{ml}$ of peptone water and left for 30 minutes (generation time of Staphylococcus aureus) to become in the log phase, the spectrophotometer was calibrated and adjusted to wave length $600 \mathrm{~nm}$, then the optical density of each of the suspensions was measured by spectrophotometer and recorded, the results were compared with standard curve that done by preparation of McFarland $0.5\left(10^{8} \mathrm{CFU} / \mathrm{ml}\right)$ that use to adjust the bacterial infection, the McFarland diluted in serial dilutions, the McFarland diluted tubes were shaked before measured by spectrophotometer at wavelength of $600 \mathrm{~nm}$. The optical densities were recorded, curve drawn from the dilutions and the optical densities.

The evaluation of the antimicrobial activity carried out by plotting the average of the optical densities of each group in the standard curve of McFarland [17].

\subsubsection{Histological Analysis}

Skin tissues were collected on day four and eight and transferred to $10 \%$ buffered formalin (BF) for two weeks. The formalin-fixed tissues were dehydrated through grades of alcohol and cleared in xylene, and then embedded in paraffin wax $\left(58-60^{\circ} \mathrm{C}\right.$ m.p.). Five to $7 \mu \mathrm{m}$ sections were deparaffinised and stained with haematoxylin and then counterstained with eosin, collagen demonstrated by Van Gieson stain [18].

\subsection{Statistical Analysis}

All results were expressed as mean $\pm \mathrm{S}$. D., and the results were compared statistically by one-way ANOVA using SPSS software and Microsoft office Excel. A p value $<0.05$ was considered statistically significant.

\section{Result}

\subsection{In Vitro Antibacterial Activity}

The antibacterial activity against the standard bacterial strains are given in table one, the different concentrations $(100,50) \%$ of the extract exhibited favourable activity with clear zone of inhibition in cup plate agar diffusion method. Staphylococcus aureus gave $17 \mathrm{~mm}$ zone of inhibition with $12.5 \%$ MIC while Escherichia coli gave the largest zone of inhibition $19 \mathrm{~mm}$ with $3.3 \%$ MIC.

\begin{tabular}{|c|c|c|c|c|c|c|c|}
\hline \multirow{2}{*}{ STD isolate } & \multicolumn{5}{|c|}{ Serial dilution of MIC } & \multirow{2}{*}{ IZD $100 \%$} & \multirow{2}{*}{ IZD $50 \%$} \\
\hline & $50 \%$ & $25 \%$ & $12.5 \%$ & $6.3 \%$ & $3.3 \%$ & & \\
\hline S.aureus & N. G & N. G & N. G & $\mathrm{G}$ & $\mathrm{G}$ & 17 & 16 \\
\hline P. mirabilis & N. G & N. G & N. G & N. G & $\mathrm{G}$ & 18 & 16 \\
\hline P.aeruginosa & N. G & N. G & N. G & N. G & $\mathrm{G}$ & 18 & 16 \\
\hline K.pneumonie & N. G & N. G & N. G & N. G & G & 17 & 16 \\
\hline E. coli & N. G & N. G & N. G & N. G & N. G & 19 & 17 \\
\hline
\end{tabular}

Table 1. Anti-bacterial activity (zone of inhibition and MIC) of Zingiber officinale extract against standard bacterial strains.

Keys

- $\mathrm{IZD}=$ Inhibition zone diameter

- $\mathrm{G} .=$ Growth

- N. G.= No growth

- Interpretation of the results:

- $\operatorname{IZD}(\mathrm{mm}):>14 \mathrm{~mm}$ : Sensitive

- <14mm: resistant

\subsection{Microbial Count Analysis}

The application of ginger extract-based ointment resulted in diminishing total bacterial counts in the infected wound, the positive control group gave significant bacterial count reduction from. $5 \times 10^{8} \mathrm{cfu} / \mathrm{ml}$ to. $5 \times 10^{3} \mathrm{cfu} / \mathrm{ml}$ ond day four, completed diminishing of bacterial infection was observed on day 8 of dressing. 
Zingiber officinale group exhibited reduction of bacterial number from. $5 \times 10^{8} \mathrm{cfu} / \mathrm{ml}$ to. $5 \times 10^{4} \mathrm{cfu} / \mathrm{ml}$ on day four and. $5 \times 10^{2} \mathrm{cfu} / \mathrm{ml}$ on day 8 of dressing, the result was close related to positive control group compared to negative control that showed reduction of bacterial from. $5 \times 10^{8} \mathrm{cfu} / \mathrm{ml}$ to. $5 \times 10^{7} \mathrm{cfu} / \mathrm{ml}$ on day 4 while in day 8 bacterial number decreased to. $5 \times 10^{6} \mathrm{cfu} / \mathrm{ml}$.

\subsection{Wound Closure Rate}

A significant difference in wound closure was observed between the groups of ginger and positive control from day 4 onwards to day 8 , the rate of wound closure group was much faster than in the negative control group (Figure 1).

\subsection{Histopathology Analysis}

Haematoxylin and Eosin stained sections of positive control and ginger groups exhibited high number of polymorphonuclear leukocyte, macrophage and moderate epithelialization on day 4, reduction of the inflammatory cell, neutrophil and macrophage were observed on day 8 due to the antimicrobial activity and more presence of epithelialization, control negative showed persistence of inflammatory cells.

Van Gieson stain gave demonstration of collagen fibers as healing process for wound; collagen fibers appeared as red colour. Significant increase of collagen fibers was observed on day 4 for both the ginger treatment group and positive control group while negative control group didn't show collagen fibers formation, on day 8 the amount of collagen increased for both ginger group and positive control group due to enhanced migration of fibroblast and epithelial cell to the wound site while the negative control group showed small amount of collagen on day 8. Ginger group exhibit more amount of collagen formation than positive control (fusiderm) on day 4.

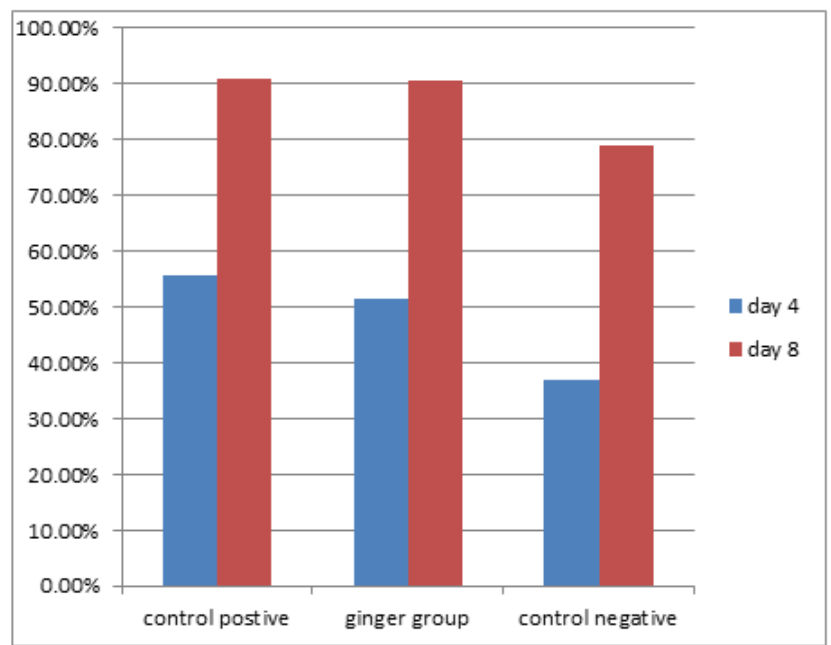

Figure 1. Percentage wound closure in the positive control, ginger and negative control groups in different time intervals (4 days and 8 days).

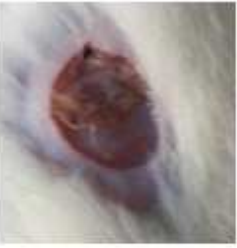

Day 0

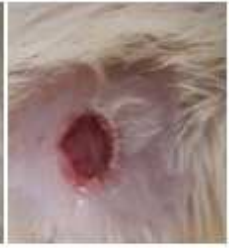

Dav4

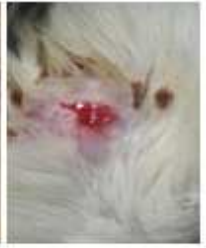

Day8
Figure 2. Photographical representation of wound contraction rate on different days in ginger treatment group.

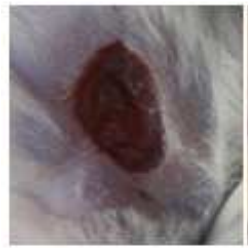

Day 0

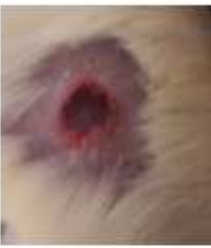

Day 4

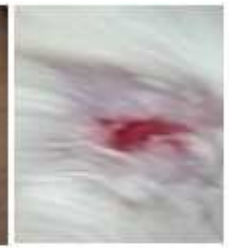

Day 8
Figure 3. Photographical representation of wound contraction rate on different days in positive control group (Fusiderm group).

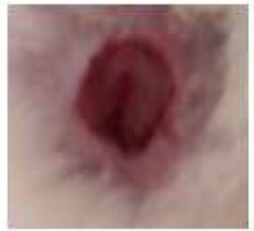

Day0

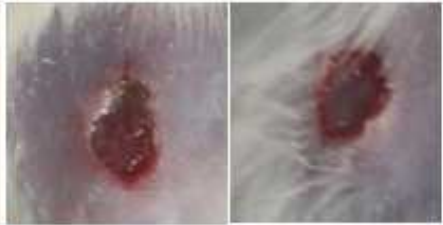

Day 4
Day 8
Figure 4. Photographical representation of wound contraction rate on different days in negative control group.

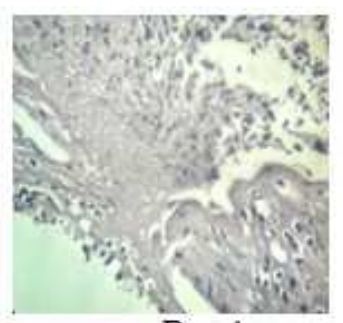

Day 4

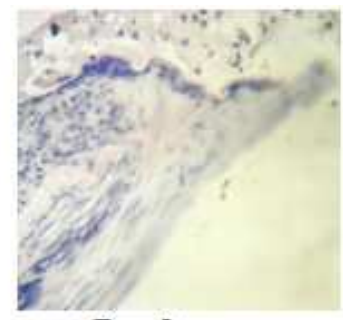

Day 8
Figure 5. Haematoxylin and eosin stained sections of the granulation tissue of positive positive treated group at different time intervals showed macrophages (M), polymorph nuclear cell (PMN) and inflammatory cell (IC).
Day4

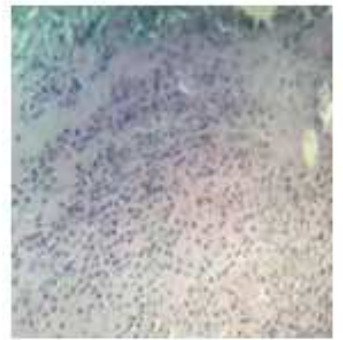

Day8

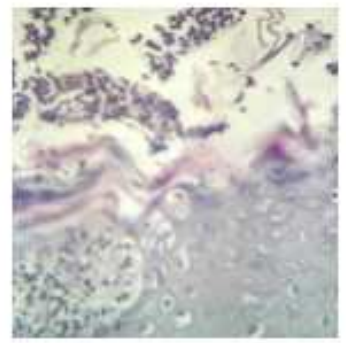

Figure 6. Haematoxylin and eosin stained sections of the granulation tissue of ginger group at different time intervals showed macrophages (M), polymorphonuclear cell (PMN) and inflammatory cell (IC). 


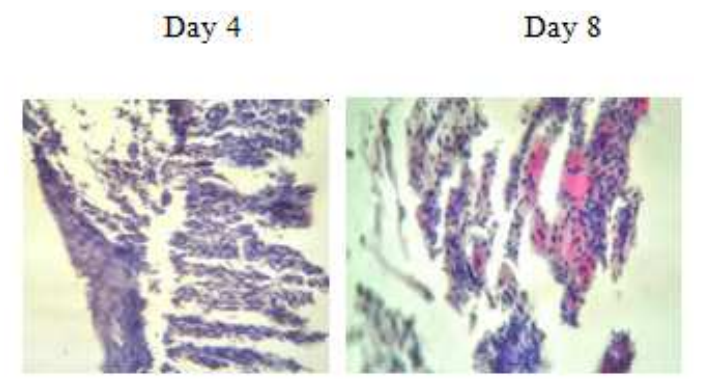

Figure 7. Haematoxylin and eosin stained sections of the granulation tissue of negative control group at different time intervals showed macrophages (M), polymorph nuclear cell (PMN) and inflammatory cells (IC).

Day 4

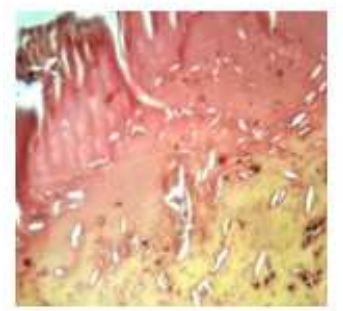

Day 8

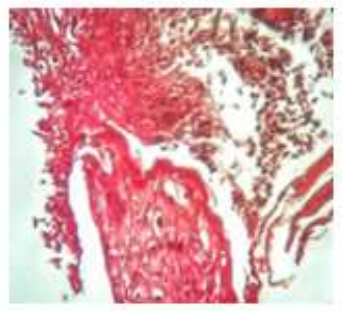

Figure 8. Van Gieson's stained sections of the granulation tissue of positive control group at different time intervals showed collagen formation appear as red colour.

Day4

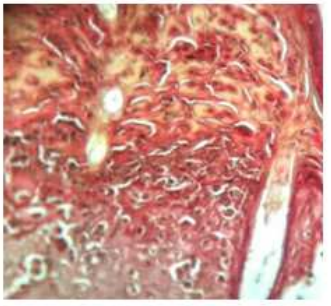

Day8

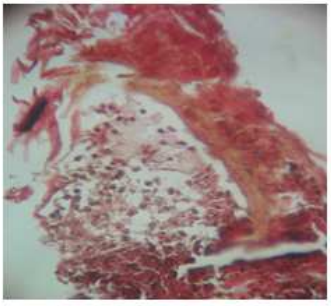

Figure 9. Van Gieson's stained sections of the granulation tissue of ginger group at different time intervals showed collagen formation appear as red colour.

\section{Day4}

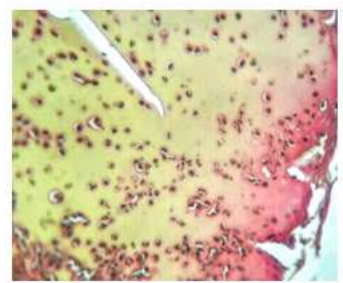

Figure 10. Van Gieson's stained sections of the granulation tissue of negative control group at different time intervals showed collagen formation appear as red colour.

\section{Discussion}

The topical application of drugs is more effective in both antimicrobial activity and wound healing rate because of its larger availability at the infected wound site. The ability of microorganisms in the wound bed to create massive damage depends on the virulence capacity of the organism and the amount of inoculum present in the wound site along with the host immune response. Staphylococcus aureus is one of the most common wound pathogens.

Sensitivity tests measure antimicrobial activity against bacteria under laboratory conditions (in vitro activity), not in the Patient (in vivo activity). It cannot be assured that an antimicrobial which kills or prevents an organism from growing in vitro will be a successful treatment in vivo so in this study in vivo antibacterial activity of Zingiber officinale plant extract was carried out in septic wound of Wistar rats which resemble human in many features.

This study agreed with Akoadere in 2002 in the result of the in vitro antibacterial effect of Zingiber officinale against Staphylococcus aureus and Proteus mirabilis, Akoadere reported that ethanolic extract of ginger had no effect against Escherichia coli but this study exhibited the largest zone of inhibition [19]. In this study, fusiderm was used as reference drug, significant reduction of bacterial number was observed in positive control, more reduction of infection on day 8 than ginger group.

Regarding the wound healing percentage of the positive control and ginger group, there were close related to each other on both day 4 and day 8 , histological examination of collagen and present of inflammatory cell agreed with wound healing percentage, both groups exhibited increasing of collagen formation and reduction of inflammatory cell from day 4 to day 8 .

This present study agreed with Roscoe 12009 study that showed the healing of abrasion wound treated with ginger extract was more rapid than negative control group rats, both of the studies showed collagen production in histological examination of skin samples that taken at time of wound healing [20].

In 2012 Lachimanan study the wound healing potential of Elaies guineensis jacq leaves the result showed better wound closure was observed in the treatment group on day 16 where it took about 25 days in negative control group [21], this present study show better wound closure of ginger almost to complete healing on day eight.

The study used size of wound of $1 \times 1 \mathrm{~cm}$ while Lachimanan study used size of wound $1.5 \times 1.5 \mathrm{~cm}$. Lachimanan study showed reduction of bacterial count on day 16 to $10^{2} \mathrm{cfu}$ in treated group compared to $10^{4} \mathrm{cfu}$ in negative control group [21]. This study show more bacterial reduction using ginger extract to $10^{2} \mathrm{cfu} / \mathrm{ml}$ on day 8 and $10^{6} \mathrm{cfu} / \mathrm{ml}$ in negative control group.

Both studies used soft yellow paraffin as ointment base for plant extract, samples were collected at 4 days interval and wounds healing measured by histological examination of Haematoxylin and Eosin stained sections of skin samples [21].

In 2008 Muthusamy studied the wound healing of triphala plant, their study was agree with our study in the use of Staphylococcus aureus to infect wounds, Muthusamy showed reduction of bacterial number from $5 \times 10$ to $37 \times 10^{2} \mathrm{cfu} / \mathrm{g}$ on day four [22], this present study showed less bacterial 
reduction of ginger treated rats from $10^{8} \mathrm{cfu} / \mathrm{ml}$ to $10^{4} \mathrm{cfu} / \mathrm{ml}$ on day four.

Both of the studies used the histological examination with Haematoxylin and Eosin stained sections, epithelialization and the reduction of inflammatory cell on day 8 in plant treated groups was much faster than negative control group [22].

\section{Conculison}

The present study concluded that ethanolic extract of Zingiber officinale in yellow soft paraffin ointment base had exhibited in vitro and in vivo antimicrobial activity against Staphylococcus aureus and wound healing potentials, also the application of ginger extract was found to improve the different phases of wound repair including collagen synthesis and maturation, wound contraction and epithelialization.

We recommended to identify the chemical constituents responsible for the antimicrobial activity of the plant and mode of action, toxicological and clinical studies should be carry out to assess the safety, therapeutic efficacy and the potential for commercial utilization.

\section{Acknowledgments}

Great thank to Allah first then to my family and Ost. Mahadi Hassan for the motivation and support throughout our study,

Our appreciation to Ost. Mahmoud and technical staff of Department of Microbiology and Histology, Al-Zaiem AlAzhari University for the kinds helps.

Thanks also to the department of veterinary, University of Khartoum and their technical staff, for the kind help through the experimental animal housing.

\section{References}

[1] Kumar, B.; Vijayakumar, M.; Govindarajan, R.; Pushpangadan, P. Ethno pharmacological approaches to wound Healing Exploring medicinal plants of India. $J$. Ethnopharmacol. 2007, 114, 103-113.

[2] Bowler PG. The anaerobic and aerobic microbiology of wounds: A review. Wounds 1998; 10: 170.

[3] Bowler PG, Davies BJ. The microbiology of acute and chronic wounds. Wounds 1999; 11: 72.

[4] Robson MC. Wound infection. A failure of wound healing caused by an imbalance of bacteria. Surg Clin N Am 1997;77: 637.

[5] Griffith-Jones A. Methicillin resistant Staphylococcus aureus in wound care. J Wound Care 1995; 4: 481.
[6] Akroum S, Satta D and Lalauoui K. (2009) Antibacterial antioxidant, cytotoxic activities and phytochemical screening of some Algerian plants. Euro. J Sci. Res., 31 (2), pp. 289-295.

[7] Mona A Mohamed. Antimicrobial agent, preparation of extract in biological activity of Nigella Sativa. Egypt, Cairo2008; page20.

[8] Singh G. Studies on the Biocidal activities of certain essential oils. Journal of Medicinal and Aromatic plant Sciences 1999; 21 (4) 1119-1123.

[9] Tan B. K. H and Vanitha J; Immunomodulatory and Antimicrobial Effects of Some Traditional Chinese Medicinal Herbs; A Review, Current Medicinal Chemistry, Vol. 11, No. 11, 2004, pp. 1423-1430.

[10] Zaika L L; "Spices and Herbs. Their antimicrobial activity and its determination;" Journal of Food Safety, Vol. 9, No. 21, 1975, pp. 97-118.

[11] Opdyke DL J; Food Cosmet. Toxicology 1974; 12 (Suppl.) PP. 901.

[12] Gao D, Zhang Y. Comparative antibacterial activities of crude polysaccharides and flavonoids from Zingiber officinale and their extraction. Asian j Trad Med.2010; 5: 235-238.

[13] Cooper K E and Kavanagh. F. (ED). Analytical microbiology. Academic Press, New York Vol. 1. 1972.

[14] Tilton R C and Howard B J. Antimicrobial susceptibility testing In Carson D, Birchor S, Clinical and pathogenic Microbiology. Mosby Co, St Louis, pp 121_115.

[15] Carter, S. L. Cooper and Gunn's Dispensing for Pharmaceutical Students; CBS Publisher and Distributors: Delhi, India, 1997; pp. 199-200.

[16] Abd Allah, A. N. (2004). Antimicrobial and wound healing activity of ten selected medicinal plants. unbuplished.

[17] European journal of applied microbiology and biotechnology Volume 9, Issue 1, pp 59-62.

[18] Mcmanus, J. G. A.; Mowry, R. W. Staining Methods: Histological and Histochemical; Harper and Row: New York, NY, USA, 1984.

[19] Ekwenye U N, department of microbiology; Michael Okapra University of agriculture, Umudike. P. M. B 7267 Umuahia, Abia state. Nigera, international journal of molecular medicine and advance science 2005; 1 (4): 416.

[20] Roscoe L. Warner, a combination of curcumin and ginger extract improves abrasion wound healing of corticosteroid damaged hairless rat skin, Wound Repair Regen. 2009; 17 (3): 360 .

[21] Sreenivasan Sasidharan, Wound Healing Potential of Elaeis guineensis Jacq Leaves in an Infected Albino Rat Mode, Molecules 2010, 15, 3186-3199.

[22] Muthusamy S Kumar 2008, Triphala Promotes Healing of Infected Full-Thickness Dermal Wound, Journal of Surgical Research 144, 94-101. 